\title{
Wavelength converter using a highly Er-doped optical fiber ring laser
}

\author{
R. A. Perez-Herrera, M. Lopez-Amo, Senior Member, IEEE, L. Rodriguez, D. Ventura, J. M. Lopez- \\ Higuera, Senior Member, IEEE
}

\begin{abstract}
This work presents an all-fiber wavelength converter based on a highly Er-doped fiber laser which has been experimentally demonstrated. Frequencies from several kilohertz up to tens of gigahertz have been investigated. Making use of the gain competition in the amplifying medium, the wavelength conversion can be carried out over not only one lasing wavelength but over several ones. These analyses have been also carried out for more than one all-fiber ring structure, demonstrating the viability of this wavelength converter. To the best of our knowledge, this is the first time an Er-doped fiber wavelength converter has been validated.
\end{abstract}

Index Terms - Fiber optics communications, intensity modulation, multiplexing, wavelength converter, optical communication, fiber optic sensors.

\section{INTRODUCTION}

$\mathrm{W}$ AVELENGTH converter (WC) is one of the key components for wavelength-division-multiplexing optical networks and photonic switch blocks [1].

Wavelength conversion is an important technique for efficient use of wavelength resources in WDM optical networks and there are many methods for achieving the wavelength conversion. For example, by using optoelectronic $(\mathrm{O} / \mathrm{E} / \mathrm{O})$ conversion, or optical gating, or four wave mixing (FWM) [2]. In addition to this, all optical wavelength converters are expected to become a key component in the future optical communication systems as well as in optical sensor networks [3], [4].

There are a number of all-fiber wavelength converters based on FWM in use today; however they present several disadvantages such as the crosstalk noise produced by the satellite signal [5]-[6], the need of highly nonlinear optical fibers (HNLF) [7]-[9] or their level of complexity [10].

In this paper a new wavelength conversion technique based on a single-mode multiwavelength lasing structure is shown. This laser, which can be operated in single-mode regime [11], is a simple structure which uses a highly Er-doped fiber.

In spite of higher pumping requirements, high concentration EDFAs having short doped fiber lengths offer a significant

Manuscript received June, 2017. This work was supported by the Spanish Comisión Interministerial de Ciencia y Tecnología (CICYT TEC2013-47264C2 and TEC2016-76021-C2) and FEDER Funds. We thank Dr. M.A. Quintela and R. Ruiz for their help with the experiment.

R.A. Perez-Herrera, M. Lopez-Amo and D. Ventura are with the Department of Electric and Electronic Engineering and ISC, Universidad Pública de advantage of compactness for fiber lasers. Most important, for high speed (or high frequency) fiber laser applications, short and high gain EDF lengths are needed [12].

This laser is a well suited candidate for wavelength conversion. The reason is the homogeneous and inhomogeneous gain broadening observed in EDFAs [13]. This effect can be used as cross-gain mechanism as in semiconductor optical amplifiers [14]. An intensity modulated input signal modulates the gain of the EDFA due to gain saturation. Thus this modulated gain can affect to the amplitude of another nonmodulated signal present in the same EDFA. This non-desired effect has been used in our structure to transfer the information from an input wavelength to another generated by the lasing structure. The converter could be used in nodes of high speed transport optical communication systems, or trunks, which are based on time division multiplexing (TDM). Therefore it would be possible to apply this technique to convert amplitude modulated signals at different wavelengths in such systems.

So for the first time a wavelength converter based on a highly-doped erbium fiber ring laser has been experimentally developed and tested. In this work, frequencies from hundreds of kilohertz up to tens of gigahertz have been investigated.

These analyses have been also carried out for more than one unmodulated lasing wavelength in order to demonstrate the viability of this technology based on the mode competition effect.

\section{EXPERIMENTAL SETUP}

\section{A. Low frequencies, from $2 \mathrm{KHz}$ to around $500 \mathrm{KHz}$}

The first schematic setup of the Wavelength Converter (WC) erbium doped fiber ring laser (EDFRL) used to carry out this experiment is shown in Fig. 1. This initial WC fiber ring laser comprises a standard single-mode fiber (SMF) ring within which a highly erbium doped fiber (M-12, with absorption of $16-20 \mathrm{~dB} / \mathrm{m}$ at $1531 \mathrm{~nm}$, by Fibercore) has been incorporated, acting as the active medium. The amplification was obtained by using about 4 meters of this highly erbium doped fiber. The fiber length needed for the cavity was shorter than in other cases [15]. Moreover, a wavelength division multiplexer (WDM) and

Navarra, Campus Arrosadía s/n E-31006 Pamplona, Spain (corresponding author to provide phone: +34948 169328; fax: +34948 169720; e-mail: rosa.perez@unavarra.es; mla@unavarra.es; ventura.60564@e.unavarra.es).

L. Rodriguez, and J.M. Lopez-Higuera are with the Photonics Engineering Group, University of Cantabria, Cantabria, Spain (e-mail: luis.rodriguez@unican.es; higuera@teisa.unican.es). 
a 980 -nm pump source were used to form the ring resonator. To ensure unidirectional oscillation in the ring cavity avoiding the hole burning effect an optical isolator was also included.

The wavelength selection was carried out by means of several FBGs and a $3 \mathrm{~dB}$ coupler was used to incorporate the FBGs into the laser cavity. Between the coupler and one of the FBGs, a four port optical circulator was placed in order to insert polarization controller (PC) and an amplitude modulator (MOD). This MOD was connected to a signal generator in order to sweep different ranges of frequencies. Finally, a $3 \mathrm{~dB}$ coupler was employed to extract part of the erbium doped fiber laser optical power from the ring to the output port to be measured by using different devices depending on the selected frequency range. In this experimental demonstration, the attained reflected signal from one of the FBGs was externally modulated at different frequencies in order to demonstrate its effect on the other lasing wavelengths. In this work, frequencies from several kilohertz up to tens of gigahertz have been investigated.

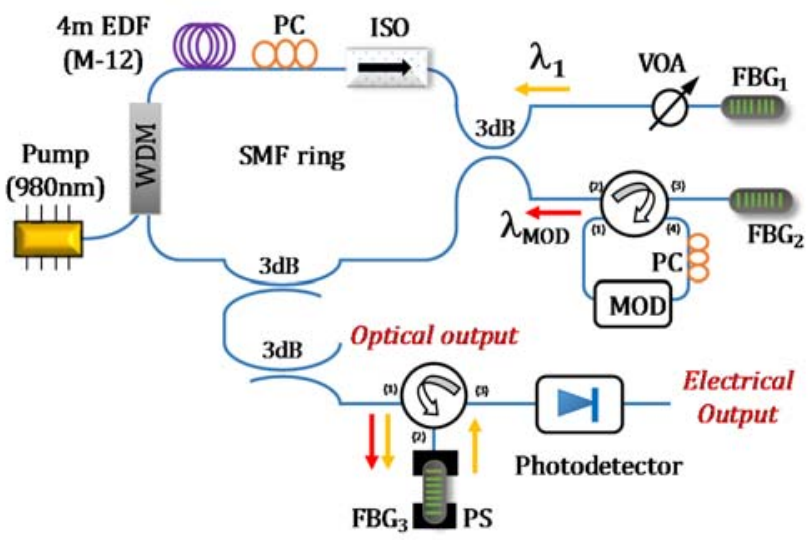

Fig. 1. Schematic of the WC-EDFRL based on optical couplers. MOD: intensity modulator; ISO: optical isolator; PS: stress inducing plate; PC: polarization controller, VOA: variable optical attenuator.

In this experiment the used FBGs were centered at $1550 \mathrm{~nm}$ and $1554 \mathrm{~nm}$ for $\mathrm{FBG}_{1}$ and $\mathrm{FBG}_{2}$ in that order. The last $\mathrm{FBG}$, centered at about $1553.9 \mathrm{~nm}\left(\mathrm{FBG}_{3}\right)$, was glued to two stress inducing plates (SP) and stretched using a motor stage (Newport MM4005), achieving very precise control of its Bragg wavelength in order to maintain its wavelength emission at $1554 \mathrm{~nm}$ (the same as $\mathrm{FBG}_{1}$ ) and to eliminate the externally modulated lasing wavelength, centered at $1550 \mathrm{~nm}$. The microdisplacements applied to the SP could be controlled by a high precision motorized stage in order to modify their lasing wavelength emission to be used in combination with another FBGs. In this first case, the amplitude modulator was tuned from several $\mathrm{KHz}$ up to about $500 \mathrm{KHz}$.

From now on, the lasing modulated and unmodulated wavelengths will be named as $\lambda_{\text {MOD }}$ and $\lambda_{\text {UNMOD }}$ respectively.

The output signal was measured by using an optical spectrum analyzer (OSA) in order to control the output power level of the generated lasing wavelengths and to equalize both signals by means of a variable optical attenuator (VOA) if required. Simultaneously, and after filtering the signal coming from $\lambda_{\text {MOD, }}$, the unmodulated lasing wavelength ( $\left.\lambda_{\text {UNMOD }}\right)$ was monitored by an electrical spectrum analyzer (ESA), whose resolution bandwidth can be as good as $1 \mathrm{~Hz}$, through the use of a photodetector.

\section{B. High frequencies, from $4 \mathrm{GHz}$ to around $20 \mathrm{GHz}$}

After demonstrating the viability of this experiment for a range of low frequencies, the external intensity modulator was replaced by another one which allowed to modulate lasing wavelengths at higher frequencies. Because of that, the optical output could be simultaneously measured by means of the OSA and a high-resolution optical spectrum analyzer (BOSA-C). This device offers a high resolution $(0.08 \mathrm{pm})$ and a high dynamic range $(>80 \mathrm{~dB})$ at the same time, avoiding the need to use neither the $\mathrm{FBG}_{3}$ nor the photodetector. In this section, frequencies from $4 \mathrm{GHz}$ to $20 \mathrm{GHz}$ have been explored in order to study this wavelength conversion at higher frequencies. Moreover, a new unmodulated lasing wavelength centered at $1548.5 \mathrm{~nm}$ was inserted into the cavity ring to experimentally demonstrate the wavelength conversion over more than one lasing wavelength.

Apart from that, another EDFRL structure based on optical circulators to insert the FBGs reflected signals into the ring (see Figure 2) was also analyzed. The challenge was to experimentally demonstrate this wavelength conversion technique over different all-fiber structures. In this configuration, two circulators were used to direct the signal inside the ring, ensuring unidirectional operation and therefore avoiding the spatial hole-burning effect. Each branch is composed of a FBG, an optical circulator, and a VOA. The intensity modulator together with the PC were inserted between the output port of one of the optical circulators and the optical coupler used to extract $10 \%$ of the laser output power from the ring. After that this extracted signal was measured by means of an OSA and a BOSA simultaneously.

As it was previously proposed by the authors [16], using this configuration, a better stability both in emission power and wavelength were obtained when compare with the one presented in Figure 1.

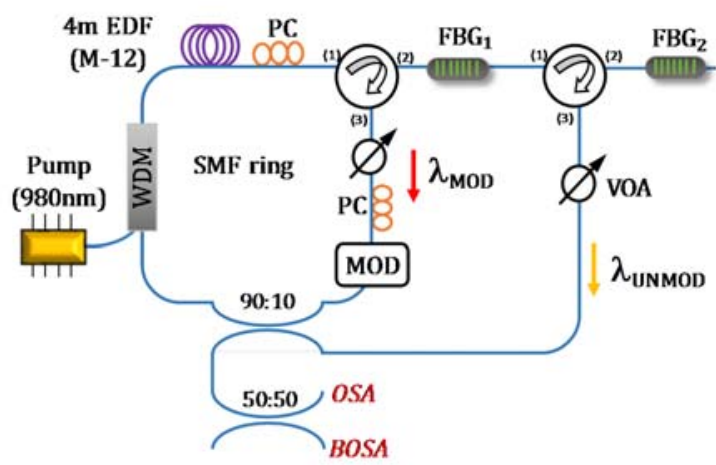

Fig. 2. Schematic of the WC-EDFRL based on optical circulators.

\section{RESULtS}

The optical spectrum of the first experimental setup when a pump power of about $110 \mathrm{~mW}$ and an intensity modulation of $2 \mathrm{KHz}$ were applied is shown in Figure 3. The power of each of 
the two output channels was around $-27.5 \mathrm{dBm}$ and $-29.4 \mathrm{dBm}$, respectively. For every channel, the signal power was about 50 $\mathrm{dB}$ higher than the ASE noise floor.

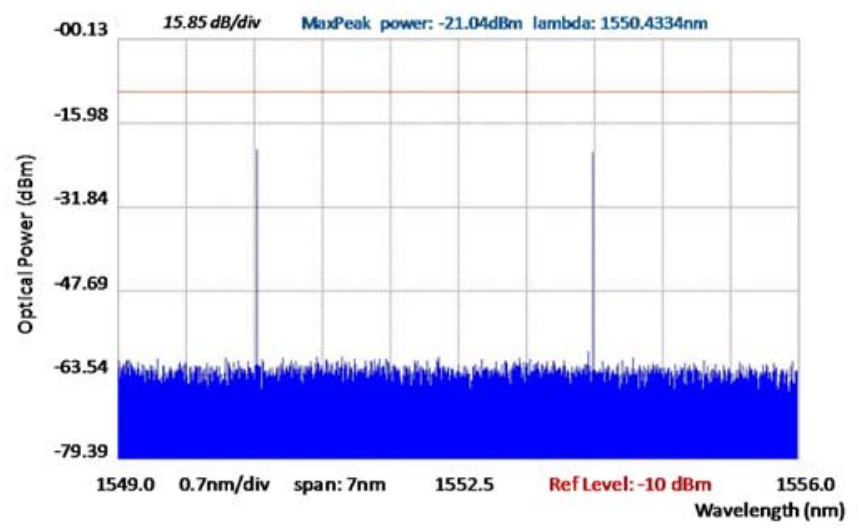

Fig. 3. Output spectrum of the EDFRL when an intensity modulation of $2 \mathrm{KHz}$ was applied to the lasing wavelength centered at $1554 \mathrm{~nm}$ measured by a BOSA.

This work experimentally demonstrates a wavelength conversion based on the gain competition between the lasing wavelengths. Because of that, these two lasers were deliberately not equalized with the purpose of increasing this effect. As can be seen in figure 4, a superimposed ripple appears over both lasers even when only one of them has been externally modulated.

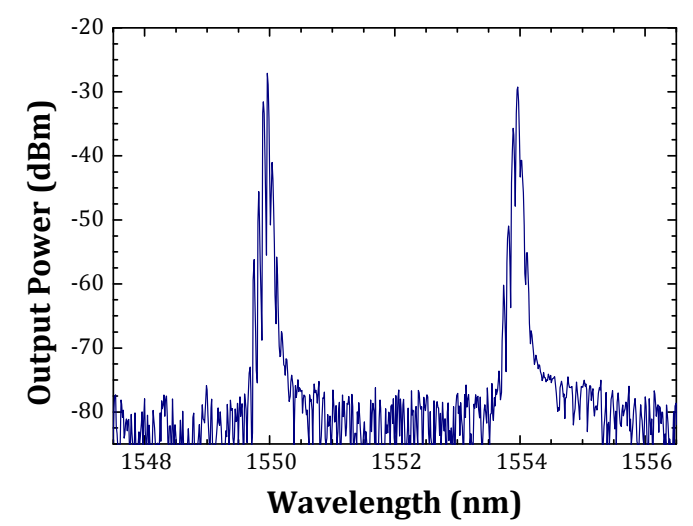

Fig. 4. Output spectrum of the EDFRL when an intensity modulation of $2 \mathrm{KHz}$ was applied to the lasing wavelength centered at $1554 \mathrm{~nm}$ measured by an OSA.

Figure 5 illustrates the output power variations measured by an OSA with a $0 \mathrm{~nm}$-span centered at $\lambda_{\text {UNMOD }}$ when a sinusoidal (Fig 4. a), triangular (Fig 4. b) or square (Fig 4. c) modulation at $500 \mathrm{~Hz}$ were applied to $\lambda_{\text {MOD. }}$. As can be observed in these three figures, the amplitude modulation applied to $\lambda_{\text {MOD }}$ can be also observed at $\lambda_{\text {UNMOD. }}$.
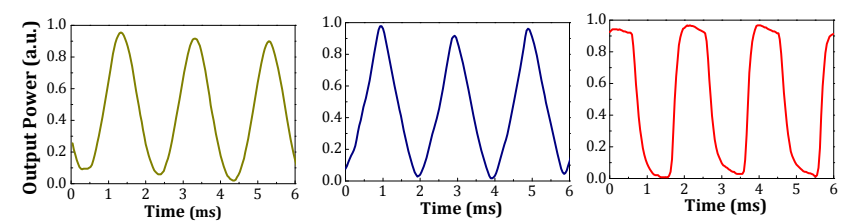

Fig. 5. Laser modulation measured at $\lambda_{\text {UNMOD }}$ when a sinusoidal (a), triangular (b) or square (c) modulation at $500 \mathrm{~Hz}$ was applied to $\lambda_{\mathrm{MOD}}$.

To analyze this effect in more detail, the electrical spectrum behavior for an intensity modulation from $100 \mathrm{KHz}$ up to 500 $\mathrm{KHz}$ was measured by means of an ESA. Figure 6 illustrates these experimental results and shows how the intensity modulation applied to $\lambda_{\text {MOD }}$ can be also measured over $\lambda_{\text {UNMOD }}$ after filtering the externally modulated lasing wavelength.

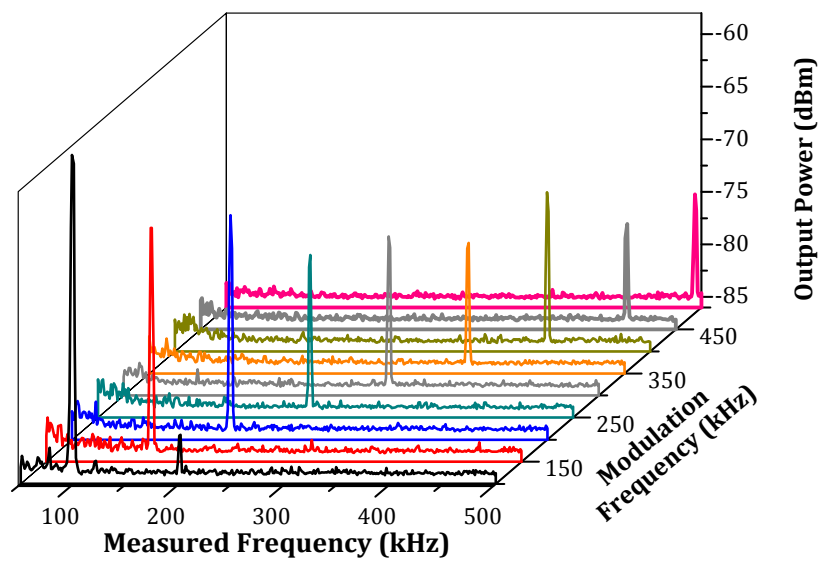

Fig. 6. Electrical spectrum behavior for intensity modulations from 100 $\mathrm{KHz}$ to $490 \mathrm{KHz}$ measured by means of an ESA after removing $\lambda_{\mathrm{MOD}}$ with a filter.

The next step consisted of increasing the modulation frequency. The intensity modulator was replaced by another with an operation range from $4 \mathrm{GHz}$ to $12 \mathrm{GHz}$. In this case, the FBGs used for the modulated and unmodulated lasing wavelengths were centered at $1552.2 \mathrm{~nm}$ and $1550.4 \mathrm{~nm}$ in that order. Figure 7 shows the optical spectrum measured by the BOSA when an intensity modulation of $5 \mathrm{GHz}$ was applied to the lasing wavelength at $1552.2 \mathrm{~nm}\left(\lambda_{\mathrm{MOD}}\right)$. Both $\lambda_{\mathrm{MOD}}$ and $\lambda_{\text {UNMOD }}$ showed only one of their sidebands. As Figure 7 shows, both lasers present exactly the same distance from their central wavelength emissions to one of their sidebands, $40.1 \mathrm{pm}$, which corresponds to a modulation frequency of $5 \mathrm{GHz}$.

In this proposed scheme, the wavelength conversion span can be tuned only by varying the modulation frequency applied into the intensity modulator, which means a remarkable improvement when compare with previous works [8].

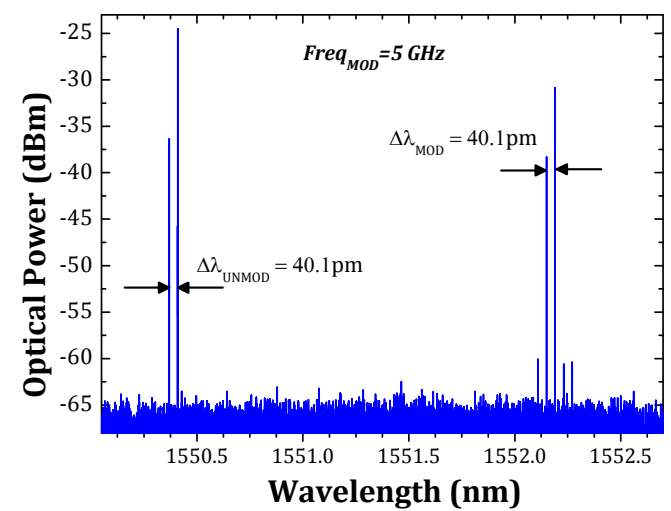


Fig. 7. Output spectrum measured by the BOSA when an amplitude modulation of $5 \mathrm{GHz}$ was applied to the lasing wavelength centered at $1552.2 \mathrm{~nm}$.

Varying the PC position these sidebands could appear or not due to the strong dependence between modulation and polarization. Here, the PC where positioned to get a single sideband modulation in order to simplify the experiment. These variations in the PC position can be also employed to attain a higher conversion efficiency of wavelength converter, which is defined as the power of converter Pout to the input Ps. Selecting a PC position whereby the single sideband was as high as possible and the central lasing wavelength disappeared, the conversion efficiency will be maximum.

The power of each of the two output channels was around $25 \mathrm{dBm}$ and $-30 \mathrm{dBm}$ for the first and second channel respectively and the optical signal to noise ratio (OSNR) was about 40 and $35 \mathrm{~dB}$ in that order, as can be seen in Figure 7. These values involve a remarkable improvement when compare with previous works [7]. In this case, the conversion efficiency was around $-10 \mathrm{~dB}$ but this value could be easily increased by varying the $\mathrm{PC}$ position. The conversion wavelength was as narrow as a single-longitudinal mode (SLM) laser behavior was attained.

This study was also repeated for a range of modulation frequencies from 4 to $12 \mathrm{GHz}$. Figure 8 shows how as the modulation frequency applied at $\lambda_{\mathrm{MOD}}$ increases the measured modulation frequency at $\lambda_{\mathrm{UNMOD}}$ equally varies.

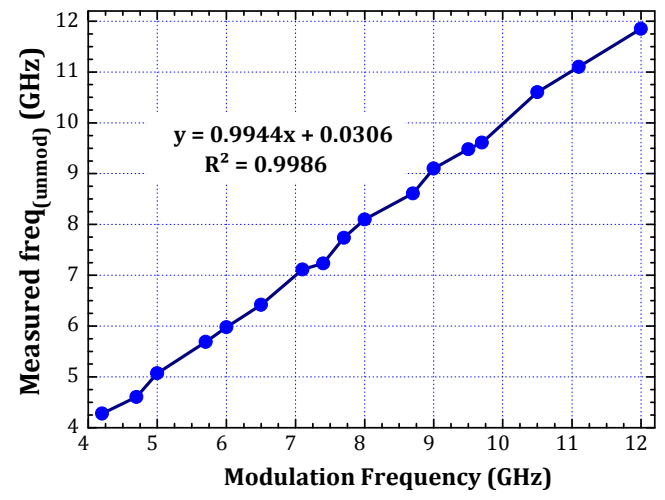

Fig. 8. Modulation frequency shift measured in the unmodulated lasing wavelength $\left(\lambda_{\mathrm{UNMOD}}\right)$ vs the modulation frequency shift applied at $\lambda_{\text {MOD }}$.

A similar analysis has been also carried out for more than one unmodulated lasing wavelength in order to demonstrate the viability of this method based on the mode competition effect to convert multiple wavelengths simultaneously. Another FBG, centered at $1548.5 \mathrm{~nm}$ was inserted into de cavity ring by means of a $3 \mathrm{~dB}$ coupler. As Figure 9 reveals, the two unmodulated lasing wavelengths, centered at $1548.5 \mathrm{~nm}$ and $1550.4 \mathrm{~nm}$, present the same distance from their central wavelength emissions to one of its sidebands, which corresponds to the modulation frequency of $5 \mathrm{GHz}$ applied to the one centered at $1552.2 \mathrm{~nm}$. The measured OSNR was around $45 \mathrm{~dB}$ for the three output channels. The conversion efficiency was around $-10 \mathrm{~dB}$ and $-20 \mathrm{~dB}$ for the first and second unmodulated lasing wavelengths in this case, being easily increased only by varying the $\mathrm{PC}$ position.

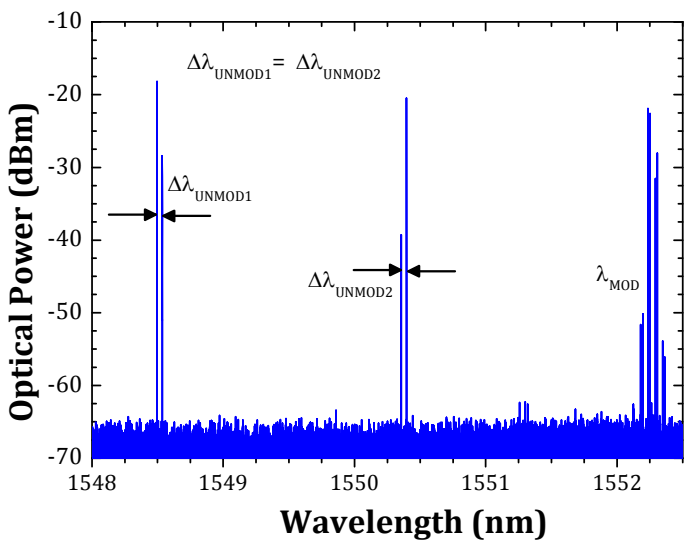

Fig. 9. Output spectrum when an amplitude modulation of $5 \mathrm{GHz}$ was applied to $\lambda_{\mathrm{MOD}}(1552.2 \mathrm{~nm})$ and two unmodulated lasing wavelengths are affected by the gain competition effect.

As in the previous case, not only an amplitude modulation of $5 \mathrm{GHz}$ was analyzed but a range of modulation frequencies were applied to this setup. The results were very similar to those shown in Figure 8 where the same modulation applied to one lasing wavelength arises over the rest of the unmodulated lasing wavelengths.

This process was also conducted for higher ranges of modulation frequencies. Afresh the intensity modulator was replaced for another with an operation range, this time from 16 $\mathrm{GHz}$ to $20 \mathrm{GHz}$. For this study, the unmodulated lasing wavelength was located closer to the modulated one; in particular it was centered at $1553.5 \mathrm{~nm}$.

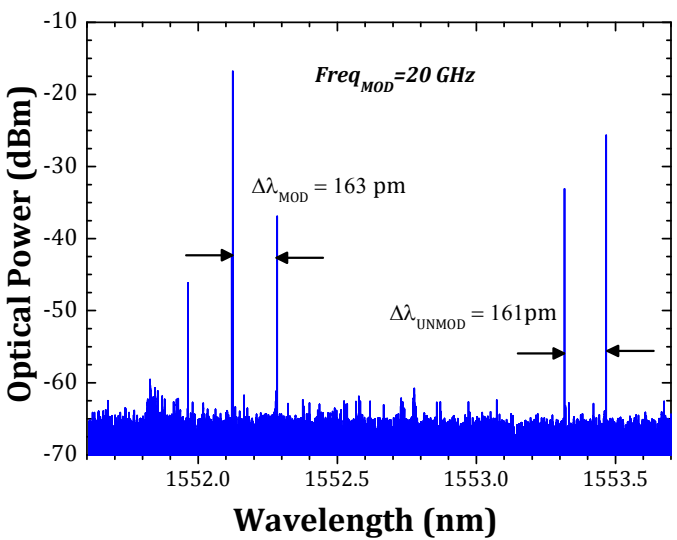

Fig. 10. Output spectrum when an amplitude modulation of $20 \mathrm{GHz}$ was applied to the lasing wavelength centered at $1552.2 \mathrm{~nm}$.

As Figure 10 shows, both lasers present the same distance from their central wavelength emissions to one of their sidebands, about $0.16 \mathrm{~nm}$, which corresponds to a modulation frequency of $20 \mathrm{GHz}$. The measured OSNR was around $45 \mathrm{~dB}$ and $40 \mathrm{~dB}$ for the first and second output channels respectively and the conversion efficiency of the unmodulated lasing wavelength was as good as $-8 \mathrm{~dB}$. 


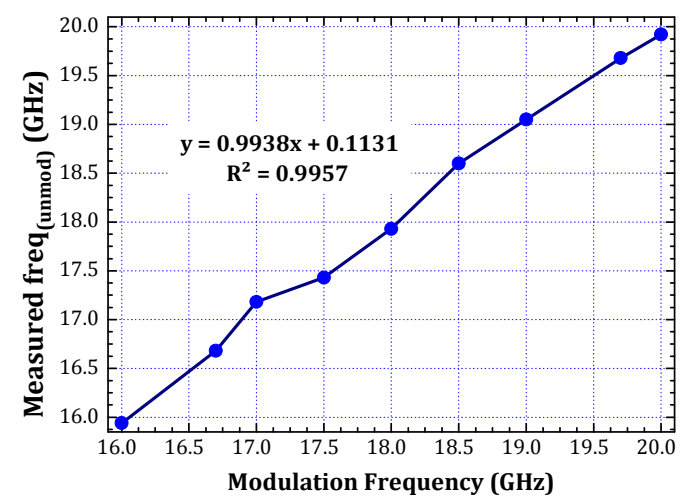

Fig. 11. Modulation frequency shift measured at $\lambda_{\text {UNMOD }}$ against the modulation frequency shift applied to $\lambda_{\text {MOD }}$.

This analysis was also repeated for a range of modulation frequencies from 12 to $20 \mathrm{GHz}$. Figure 11 shows the linear behavior of the measured modulation frequency at $\lambda_{\text {UNMOD }}$ as a function of the applied modulation frequency at $\lambda_{\mathrm{MOD}}$.

As it was previously pointed out and, with the challenge to experimentally demonstrate the behavior of this wavelength conversion, another EDFRL was also analyzed (see Figure 2). This structure was not based on couplers but based on optical circulator. Previous works for the authors [16] pointed out the advantages of this topology when compare with the one presented in Figure 1. The use of optical circulators also abolishes the need of optical isolator due to the fact that they avoid the hole burning effect in the EDFRL.

As Figure 12 indicates, this wavelength conversion was also produced when a circulator's based EDFRL is employed. The lasing wavelength at $1552.1 \mathrm{~nm}$ was externally modulated at a modulation frequency of $16 \mathrm{GHz}$ but not the one centered at $1553.4 \mathrm{~nm}$. Likewise, both sidebands appear for these two lasing wavelengths. The distance between the central emission wavelength and one of its sidebands is about $127 \mathrm{pm}$, which corresponds to a modulation frequency of $16 \mathrm{GHz}$.

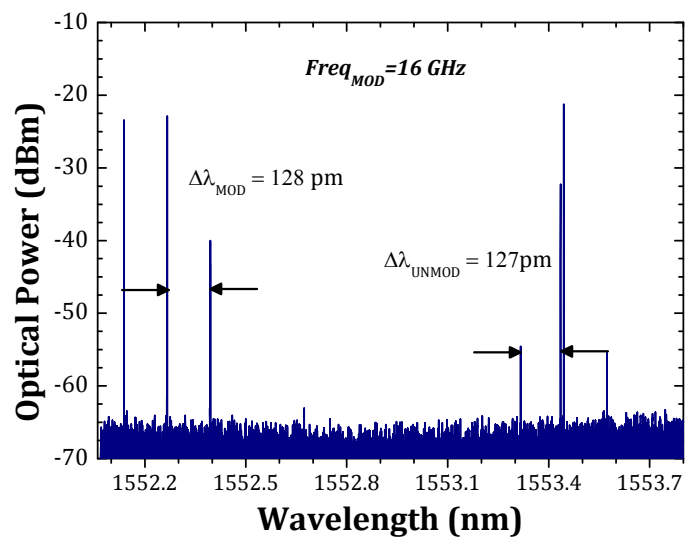

Fig. 12. Output spectrum of the EDFRL based on optical circulators when an amplitude modulation of $16 \mathrm{GHz}$ was applied to the lasing wavelength centered at $1552.2 \mathrm{~nm}$.

\section{CONCLUSIONS}

This work has detailed and experimentally demonstrated an ultra-narrow, highly efficient, and all-fiber wavelength converter based on gain competition on an Er-doped fiber laser. Frequencies from several kilohertz up to tens of gigahertz have been investigated. Making use of this competition effect, the wavelength conversion has been carried out over not only one lasing wavelength but over several ones, demonstrating that this effect can be used for the wavelength conversion of multiple lasing wavelengths simultaneously. The conversion efficiency can be easily maximized only by selecting a PC position whereby the single sideband was as high as possible and the central lasing wavelength as low as possible or even disappeared. Further research will be carried about the phase response of the optical conversion. To the best of our knowledge, this is the first time a highly Er-doped fiber laser wavelength converter has been experimentally demonstrated.

\section{REFERENCES}

[1] C. Kachris, and I. Tomkos, "A survey on optical interconnects for data center," IEEE Communications Surveys and Tutorials, vol. 14, no. 4, pp. 1021-1036, 2012

[2] M. Badar, H. Kobayasi, and K. Iwashita "Demonstration of format free wavelength conversion for optical network," Opt. Quant Electron, vol. 48, no. 1, pp. 1-9, Jan. 2016

[3] A. Kaur, K. Singh, and B. Utreja "Wavelength Converters in Optical Communication Systems," IRACST-Engineering Science and Technology: An International Journal (ESTIJ), vol.3, no.2, pp. 338-344, April 2013.

[4] "Realizing device of large-capacitance self-healing optical fiber sensing network based on light spectrum type optical fiber sensor and realizing method" Patent CN 101867506 B

[5] K.E. Stubkjaer, C. Joergensen, S.L. Danielsen, et al., "Wavelength conversion devices and techniques" in European Conference on Optical Communication, ECOC, 4, pp. 33-40 (1996)

[6] D.F. Geraghty, R.B. Lee, M. Verdiell, et al., "Wavelength conversion for WDM communication systems using four-wave mixing in semiconductor optical amplifiers," IEEE Journal on Selected Topics in Quantum Electronics, vol. 3, no. 5, pp. 1146-1155, 1997.

[7] O. M. Kharraz, H. Ahmad, D. I. Forsyth, M. Dernaika, M. Z. B. Zulkifli, M. F. B. Ismail, and A. B. B. Mohammad, "All-incoherent wavelength conversion in highly nonlinear fiber using four-wave mixing," Opt. Eng., vol. 53, no. 9, 096112, Sept. 2014

[8] O.M. Kharraz, H. Ahmad, A.B.B. Mohammad, D.I. Forsyth, M. Dernaika, and S.W. Harun, "Performance enhancement of pre-spectrum slicing technique for wavelength conversion," Opt. Comm., vol. 350, no. 1, pp. 154-159, Sept. 2015

[9] G. Shiming, Y. Changxi, X. Xiaosheng, T. Yu, Y. Zheng, and J. Guofan, "Wavelength conversion of spectrum-sliced broadband amplified spontaneous emission light by hybrid four-wave mixing in highly nonlinear, dispersion-shifted fibers," Opt. Express, vol. 14, no. 7, pp. 2873-2879, Apr. 2006

[10] H. Nguyen Tan, T. Inoue, K. Tanizawa,S. Namiki, S. Petit, Y. Oikawa, S. Takasaka, and T. Yagi, "Development of highly cascadable wavelength converter for all-optical networks," in International Conf. on Advanced Techn. for Communications (ATC 2014), Hanoi, 2014, pp. 754-759

[11] M.A. Quintela, R.A. Perez-Herrera, I. Canales, M. Fernandez-Vallejo, M. Lopez-Amo, and J.M. Lopez-Higuera, "Stabilization of dualwavelength erbium doped ring fiber lasers by single-mode operation," IEEE Photonics Techn. Lett., vol. 22, no. 6, pp. 368-370, Mar. 2010

[12] E. Desurvire, "Erbium-doped fiber amplifiers. Principles and applications" pp.388, Ed. John Wiley and Sons. New York, USA, 1994

[13] E. Desurvire "Rare-earth-doped fiber lasers and amplifiers" 2nd ed. Ed. CRC press USA pp.553-561, 2001

[14] B. Ramamurty, and B. Mukherjee "Wavelength conversion in WDM networking," IEEE J. Selected Areas in Communications, vol. 16, no. 7, pp. 1061-1073, Sept. 1998 
[15] L Talaverano, S Abad, S Jarabo, and M Lopez-Amo, "Multiwavelength fiber laser sources with Bragg-grating sensor multiplexing capability," IEEE J. of Lightwave Techn., vol.19, no. 4, pp. 553-558, Apr. 2001

[16] R.A. Perez-Herrera, M.A. Quintela, M. Fernández-Vallejo, A. Quintela, M. Lopez-Amo and J.M. Lopez-Higuera, "Stability comparison of two ring resonator structures for multiwavelength fiber lasers using highly doped Er-fibers," IEEE J. of Lightwave Techn., vol. 27, no. 14, pp. 25632569, Jul. 2009 